\title{
Sensitivity and Specificity of Computed Tomography in the Evaluation of Bone Mineral Density in Mexican Patients with Breast Cancer
}

Ana Amador Martínez ${ }^{1}$, Eleazar Lara Padilla ${ }^{2}$, Juan Antonio Pérez Rodríguez ${ }^{1}$, Alfonso Alfaro ${ }^{3}$, Dania Guadalupe Solis Cano ${ }^{1}$, Cindy Bandala ${ }^{2}$, Nancy Guzman ${ }^{1}$

1. Radiology, Centro Médico ABC, CDMX, MEX 2. Medicine, Instituto Politécnico Nacional, CDMX, MEX 3. Neuroscience, National Rehabilitation Institute, CDMX, MEX

Corresponding author: Cindy Bandala, cindimiel@hotmail.com

\section{Abstract}

\section{Introduction}

Breast cancer (BC) is the most frequently reported cancer among women - reported in 2012 as $25 \%$ of all cancers. BC has been related to the increased life and activity of osteoclasts, conferring a higher risk for osteoporosis/osteopenia. This study aimed to determine a cut-off point in Hounsfield units (HU) as well as the sensitivity and specificity of computed axial tomography (CT) in the diagnosis of osteoporosis/osteopenia in Mexican women with BC.

\section{Material and methods}

We included 108 patients with a histopathological diagnosis of $\mathrm{BC}$ treated at the $\mathrm{ABC}$ Medical Center in Mexico City. All patients were subjected to both dual X-ray densitometry and CT. The receiver operating characteristic (ROC) curve was used to identify the cutoff point and sensitivity and specificity were calculated, as were confidence intervals for the diagnoses of osteoporosis/osteopenia.

\section{Results}

The mean age was $58.49 \pm 11.01$ years. The cutoff point with the highest sensitivity $(82 \%)$ and specificity (68\%) was $<157 \mathrm{HU}$ for osteoporosis/osteopenia in patients with BC.

\section{Conclusions}

Women with $\mathrm{BC}$ are exposed to several risk factors for osteoporosis/osteopenia. The CT obtained for the general evaluation of these patients can also be used to evaluate bone mineral density, avoiding additional examinations and exposure to radiation, as well as the cost it confers, offering an earlier diagnosis of osteoporosis/osteopenia for its control.

Received 08/12/2019

Review began 08/21/2019

Review ended 08/26/2019

Published 08/28/2019

๑) Copyright 2019

Amador Martínez et al. This is an open access article distributed under the terms of the Creative Commons Attribution License CC-BY 3.0., which permits unrestricted use, distribution, and reproduction in any medium, provided the original author and source are credited.

Categories: Radiology, Oncology, Other

Keywords: bone mineral density, breast cancer, hounsfield units

\section{Introduction}

Breast cancer $(\mathrm{BC})$ is the second most common cancer in women $[1,2]$. Incidence of $\mathrm{BC}$ increases with age; it has been reported that over $75 \%$ of BC occurs in postmenopausal women [3]. Different studies show a connection between bone mineral density and BC $[4,5]$. The numerous factors favoring the appearance of osteoporosis in cancer patients are related to the tumor itself and to the antitumor treatment [6]. Chemotherapy-induced ovarian failure is a common cause of bone degradation among patients with breast cancer [7].

The recognition of osteoporosis as one of the late sequelae that may appear after the cure of cancer requires an early diagnosis of this disease in patients with an oncological background to adopt the appropriate preventive measures $[8,9]$. Bone mineral density is inversely proportional to the risk of fracture and is currently the best predictor to evaluate this parameter [10-14]. The risk of fracture for a patient with osteoporosis is $40 \%$, with the most frequent sites of this event being the spine, the hip, and the wrist, among others [15]. In addition to conventional radiography, there are other imaging techniques such as dual X-ray absorptiometry (DXA) and computed tomography quantification, which has been developed to evaluate bone mineral content [16]. The International Society for Clinical Densitometry (ISCD) recommends that the BMD (Bone Mineral Density) test should be carried out on all women 65 years of age or older, as well as all men over 70 years of age [16]. On the other hand, the Hounsfield Units (HU) represent the relative density of body tissues according to a calibrated level scale, based on values for air (-1000 HU), water (0 HU), and bone density $(+1000 \mathrm{HU})[17]$. Literature indicates that bone quality can also be determined through the 
measurements of $\mathrm{HU}$, which can be calculated from a region of interest using most modern radiology programs without additional costs or radiation [18]. A sensitivity of $86 \%$ and a specificity of $94 \%$ have been described for computed axial tomography (CT) in the diagnosis of osteoporosis [18]. DXA is currently the gold standard for assessing the bone mineral density and has been correlated with fracture risk. Although it is useful for evaluating osteopenia or osteoporosis, it poses certain methodological limitations [19]. Both DXA and CT can be used for the quantification of bone mineral density. Recently, several studies have proposed a potential correlation between BMD and the result of CT. There are different conclusions regarding the usefulness of $\mathrm{CT}$ to evaluate BMD: the majority suggests that CT provides accurate and reliable results in this tenor. CT has been suggested to have the potential to be considered an alternative method of timely detection of osteoporosis, without radiation or additional cost since CT can be analyzed for different aspects related to the patients' pathology [20-23]. Our study is a pioneer in showing the result of a CT scan as a diagnostic test for osteoporosis/osteopenia in Mexican patients with BC.

\section{Materials And Methods}

A total of 108 patients diagnosed with BC (clinical and histopathological diagnosis) - with densitometry and computed tomography studies using multidetector helical equipment, cross-sections were made with abdomen computed tomography protocol, detector coverage (mm) 40.0, helical thickness ( $\mathrm{mm}$ ) 2.5, pitch and speed (mm/rot) 1.375: 1, 55.00, rotation time (s) 0.6, mA 520, KV 120, with an approximate total dose report per patient CTD vol 11.14 (mGy), DLP $578.93(\mathrm{mGy}-\mathrm{cm})$ conducted at the ABC Cancer Center, Mexico City - were included in this study. Exclusions comprised patients with hypothyroidism, with treatments based on steroid corticosteroids, and with incomplete clinical records; unreliable DXA or CT results as patients with metallic material in vertebral bodies were also eliminated. Medical records were collected using the clinical file with prior approval from ABC Medical Center's ethics committee (folio TABC-1722). Baseline characteristics were collected from the clinical history, including data on risk and comorbidity as well as hereditary family history, smoking habits, and excess weight or obesity by body mass index. The CT scan results were collected in HU from the evaluation of the vertebral body of L3 (trabecula) using PACS. A DXA analysis was performed by an observer-blinded from the DXA results on the lumbar spine and both coxofemoral joints. Results were classified according to the World Health Organization in standard deviations (SD), according to the parameter for age in our population (Osteopenia: -1 to -2.5 SD. Osteoporosis: -2.5 SD. Normal: up to $-1 \mathrm{SD})$.

The densitometer's brand was Hologic, model Discovery WI with serial number 87039. The tomography equipment employed was a 64-slice GE CT scanner, lightspeed VCT model with serial number 5230VCTABC.

Means, standard deviations, frequencies, and percentages were determined. The diagnostic test parameters were determined for qualitative data as well as the receiver operating characteristic (ROC) curve to obtain a cut-off point in HU for osteoporosis/osteopenia. Kolmogorov-Smirnov, chi-square, ANOVA and the Pearson and Spearman correlation tests were applied. The data analysis was performed with the program SPSS v19 and Epidat 3.1. A confidence interval of $95 \%$ was taken for all tests and determinations.

\section{Results}

A total of 108 patients diagnosed with BC were studied. The average age was $58.49 \pm 11.01$ years, with a 48 years range ( 34 to 82 years). The frequencies of risk factors related to lifestyle and comorbidities were as follows: $29.6 \%$ of the patients were overweight and $16.7 \%$ were obese. The minimum BMI was $18 \mathrm{~kg} / \mathrm{m}^{2}$ and the maximum was $39 \mathrm{~kg} / \mathrm{m}^{2}$. Regarding the gynecological-obstetric history, the frequency of early menarche was $3.7 \%$ (four cases), none of the patients had late menopause and only $4.6 \%$ (five cases) was nulliparous. Stage III-IV had a frequency of $22.2 \%$ (24 cases), metastasis at the time of diagnosis was $20.4 \%$ (22 cases). Women who were in stage III-IV had 18 times more risk (95\% confidence interval of 5.83-56.14); that is, metastasis was related in $68.2 \%$ to stage III-IV ( $p=0.0001$ ). Only $9.3 \%$ (10 cases) were treated with hormone therapy and $37.4 \%$ (40 cases) with surgery. Regarding the immunohistochemical markers, $48.1 \%$ of cases had triple-negative profile (Negative estrogen receptor/negative progesterone receptor/negative HER2).

The bone density evaluated by DXA showed that $55.6 \%$ of the patients presented osteoporosis/osteopenia (45.4\% Osteopenia, 10.2\% Osteoporosis). The DXA result was related to the risk age for menopause and stage III-IV (Table 1). 


\section{Cureus}

\begin{tabular}{|c|c|c|c|c|}
\hline & \multicolumn{4}{|c|}{ DXA Study Results } \\
\hline & Normal $(n=38)$ & Osteopenia $(n=56)$ & Osteoporosis $(n=14)$ & P -value \\
\hline Age $>48$ years & $38 \%(32)$ & $50 \%(42)$ & $12 \%(10)$ & $0.04^{*}$ \\
\hline Stage III-IV & $25 \%(6)$ & $58.3 \%$ (14) & $16.7 \%$ (4) & $0.03^{\star}$ \\
\hline
\end{tabular}

\section{TABLE 1: DXA result related to the risk age for menopause and stage III-IV.}

*Statistical significance, $\mathrm{P}$-value $<0.05$, Chi square

DXA: Dual X-ray absorptiometry

Table 2 shows the HU global averages obtained through CT in relation to the qualitative result of the DXA (normal, osteopenia, osteoporosis). This same table also shows the mean HU values obtained through the CT scan in the risk age for menopause and relation to the categorical result of the DXA (normal, osteopenia, osteoporosis).

\begin{tabular}{|c|c|c|c|}
\hline \multirow[b]{2}{*}{ DXA results } & \multirow[b]{2}{*}{ Global Mean \pm SD } & \multicolumn{2}{|c|}{ Age of Risk for Menopause } \\
\hline & & $>48$ years Mean \pm SD & $<48$ years Mean \pm SD \\
\hline Normal & $187.84 \pm 64.4$ & $166.03 \pm 56.71$ & $231.46 \pm 59.4$ \\
\hline Osteopenia & $139.19 \pm 40.1$ & $134.39 \pm 38.3$ & $164.42 \pm 44.6$ \\
\hline Osteoporosis & $93.73 \pm 21.6$ & $95.74 \pm 21.73$ & 73.63 \\
\hline$P$ value & $0.0001^{*}$ & $0.0001^{*}$ & $0.007^{*}$ \\
\hline
\end{tabular}

TABLE 2: Bone density assessed by CT (HU) in relation to DXA results with the age of risk for menopause.

${ }^{*}$ Statistical significance

SD: Standard deviation; CT: Computed axial tomography; DXA: Dual X-ray absorptiometry.

Figure 1 shows the ROC curve, where the maximum point of sensitivity and specificity for CT (HU) is shown in relation to the result of osteoporosis/osteopenia according to DXA. The optimal cut-off point was $<157$ HU for osteoporosis/osteopenia in patients with BC, reaching a sensitivity of $82 \%$ and a specificity of $68 \%$, with an area under the curve of $0.76(p=0.0001)$ and a confidence interval of $95 \%$ from 0.67 to 0.86 . 


\section{Cureus}

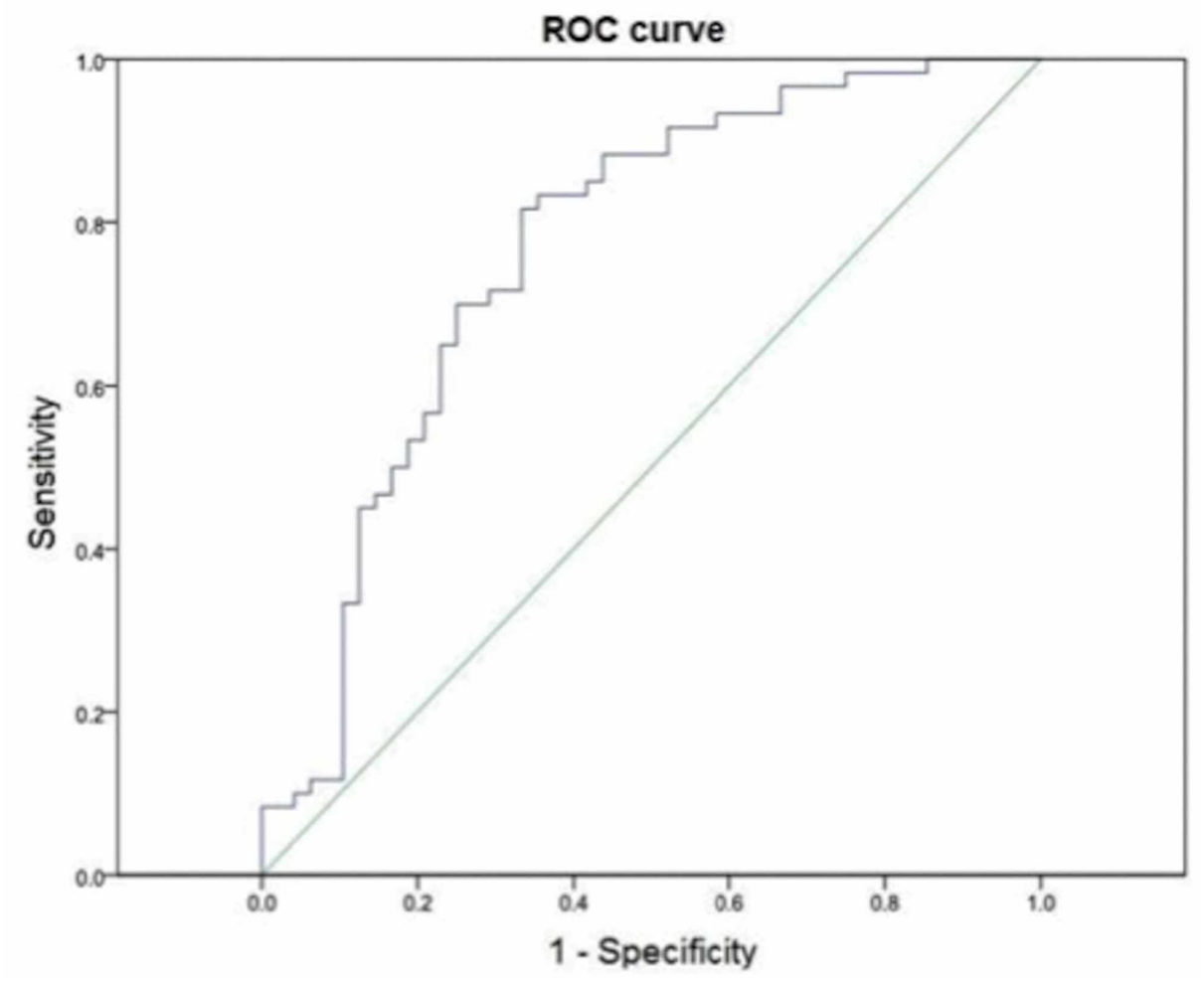

FIGURE 1: ROC curve of the CT study (HU) to evaluate bone mineral density (BMD) in patients diagnosed with BC.

ROC: Receiver operating characteristic; BC: Breast cancer.

A contingency table was made between the diagnosis of osteoporosis/osteopenia (cut-off point of $<157 \mathrm{HU}$ ) evaluated by CT and by DXA. Table 3 shows 35 cases of true positives between both diagnostic methods.

\begin{tabular}{|c|c|c|c|c|c|}
\hline & & & \multicolumn{2}{|l|}{ DXA } & \multirow{2}{*}{ Total $(n=108)$} \\
\hline & & & Osteoporosis/osteopenia $(\mathrm{N}=60)$ & Normal $(n=48)$ & \\
\hline \multirow{2}{*}{ СT } & $<157 \mathrm{HU}$ & Osteoporosis/osteopenia & $58.3 \%(35)$ & $64.6 \%(31)$ & $61.1 \%(66)$ \\
\hline & $>157 \mathrm{HU}$ & Normal & $41.7 \%(25)$ & $35.4 \%(17)$ & $38.9 \%(42)$ \\
\hline
\end{tabular}

TABLE 3: CT scan results with a cut-off point of $157 \mathrm{HU}$ in relation to the DXA results in patients with BC.

CT: Computed axial tomography; DXA: Dual X-ray absorptiometry; BC: Breast cancer.

Table 4 shows the CT scan's diagnostic test parameters, employing the cutoff point of $157 \mathrm{HU}$, and the gold standard (DXA characterized in healthy patients). 


\section{Cureus}

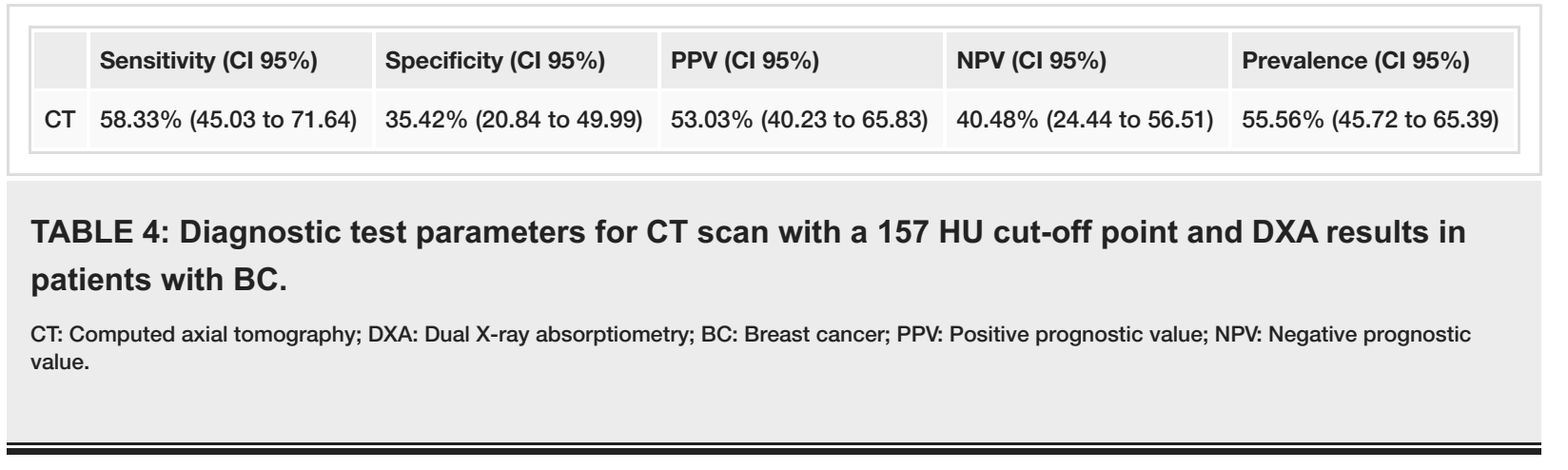

Finally, we observed that the HU obtained by CT scan negatively correlated with age $(r=-0.53, p=0.0001)$ and the DXA result $(\mathrm{r}=-0.53, \mathrm{p}=0.0001)$.

\section{Discussion}

The average age our patients presented at the time of diagnosis of BC was similar to that previously reported for the Mexican population [24-27]. Regarding excess weight and obesity, in our study, we observed lower frequencies concerning other studies conducted in our country [28]. The prevalence of osteoporosis found in our population was similar to that described in studies of osteoporosis in Europe: it was only $1.8 \%$ lower than that described in the European population [28]. According to the findings in the DXA values, the patients with osteoporosis were mostly menopausal women; when comparing clinical stages, those located in stage III-IV had the highest percentage of osteoporosis (36.4\%).

Regarding the results obtained by CT pre-menopausal patients with normal DXA presented 65.43 less HU than those found in menopausal patients with normal DXA, as expected. Likewise, when comparing the results in patients on clinical stages III-IV but with a normal DXA, these were higher (86.4 more) than that obtained in abnormal DXA patients but with early clinical stages (I-II-III). This can presumably be due to the presence of metastatic lesions as well as the biochemical processes of the activation of beta adrenergic receptors [29]. Through the CT scan, a cut-off level for osteoporosis/osteopenia equal to or $<157 \mathrm{HU}$ was obtained with a sensitivity of $82 \%$, which is very similar to that reported by Pickhardt et al. in 2013 . They reported a $90 \%$ sensitivity for a cut-off level of $160 \mathrm{HU}$, even though their study was of the North American population and included both sexes [21]. Other studies exist where authors correlate DXA with CT: Batawil and Sabiq in 2012 described a cut-off level of $203 \mathrm{HU}$ to exclude osteopenia/osteoporosis [30]. This may be because this study was conducted in the country of Saudi Arabia, with a group of women with various previous diagnoses and only some of them with BC.

According to the results from this clinical investigation, values were obtained using CT with Hounsfield units (HU) for osteoporosis/osteopenia and normal bone density. The mean for patients with BC with a normal DXA was $187.84 \mathrm{HU}$; it was $139.19 \mathrm{HU}$ for patients with a DXA showing osteopenia, and $93.73 \mathrm{HU}$ for patients with a DXA for osteoporosis. This difference in HU values was both statistically significant and similar to other studies. We conclude that computed tomography could provide a bone density profile employing Hounsfield units in all patients who undergo this study for other medical reasons. It is relevant for the clinician since the state of bone metabolism should not be forgotten in the evaluation of the patient with breast cancer, the timely detection will allow him to reduce fracture risks.

Mexican patients with breast cancer are a good example: by taking advantage of the studies that are practiced for follow-up and/or approach during clinical staging, the time-cost-radiation of dual X-ray absorptiometry can be avoided. The optimal cut-off point for osteoporosis/osteopenia through CT was equal to or less than $157 \mathrm{HU}$, reaching a sensitivity of $82 \%$ and a $68 \%$ specificity, with a $95 \%$ CI. The resulting HU correlated with age: pre-menopausal patients showed an average of $231.46 \mathrm{HU}$, unlike patients with BC and menopausal women who had an average of $166.03 \mathrm{HU}$. These results show that normal bone density parameters should be established in cancer patients with both DXA and CT studies. Normal bone density parameters should also be reestablished in the gold standard that is densitometry when it is determined in oncological patients.

\section{Limitations}

One limitation of our study was that it was not possible for us to validate our results, we only reported the descriptive findings and we proposed a cut-off point, for our population but we need to perform further studies.

\section{Conclusions}

According to the results obtained by CT in our clinical research, the average for breast cancer patients with normal densitometry was $187.84 \mathrm{HU}$, for patients with osteopenia in densitometry was $139.19 \mathrm{HU}$ and 93.73 
$\mathrm{HU}$ for osteoporotic patients by densitometry; this difference in values of $\mathrm{HU}$ was statistically significant. The optimal cut-off point obtained by ROC curve for osteoporosis/osteopenia by CT was equal or less than $157 \mathrm{HU}$, reaching a sensitivity of $82 \%$ and $68 \%$ specificity with a $95 \%$ CI. Therefore, we conclude that computed tomography can provide a bone density profile using the Hounsfield units.

\section{Additional Information \\ Disclosures}

Human subjects: All authors have confirmed that this study did not involve human participants or tissue. Animal subjects: All authors have confirmed that this study did not involve animal subjects or tissue. Conflicts of interest: In compliance with the ICMJE uniform disclosure form, all authors declare the following: Payment/services info: All authors have declared that no financial support was received from any organization for the submitted work. Financial relationships: All authors have declared that they have no financial relationships at present or within the previous three years with any organizations that might have an interest in the submitted work. Other relationships: All authors have declared that there are no other relationships or activities that could appear to have influenced the submitted work.

\section{Acknowledgements}

We would like to express our gratitude to the participants of this study. To MD Marco Antonio Téliz Meneses, Head of the Radiology and Image Department of the ABC Medical Center.

\section{References}

1. Ferlay J, Soerjomataram I, Dikshit R, et al.: Cancer incidence and mortality worldwide: sources, methods and major patterns in GLOBOCAN 2012. Int J Cancer. 2015, 136:359-386. 10.1002/ijc.29210

2. Safaei-Nodehi R, Esmaili J, Sharifian R, Movaseghi S, Parkhideh S: Does adjuvant chemotherapy change bone mineral density and related serum biomarkers in women with breast cancer?. Caspian J Intern Med. 2017, 8:91-98. 10.22088/cjim.8.2.91

3. Zain NM, Seriramulu VP, Chelliah KK: Bone mineral density and breast cancer risk factors among premenopausal and postmenopausal women - A systematic review. Asian Pac J Cancer Prev. 2016, 17:32293234.

4. Fraenkel M, Novack V, Liel Y, et al.: Association between bone mineral density and incidence of breast cancer. PLoS One. 2013, 8:70980. 10.1371/journal.pone.0070980

5. Datta M, Schwartz GG: Calcium and vitamin D supplementation and loss of bone mineral density in women undergoing breast cancer therapy. Crit Rev Oncol Hematol. 2013, 88:613-624. 10.1016/j.critrevonc.2013.07.002

6. Khosla S: Minireview: the OPG/RANKL/RANK system. Endocarinology. 2001, 142:5050-5055. 10.1210/endo.142.12.8536

7. Monroy-Cisneros K, Esparza-Romero J, Valencia ME, Guevara-Torres AG, Méndez-Estrada RO, AnduroCorona I, Astiazarán-García H: Antineoplastic treatment effect on bone mineral density in Mexican breast cancer patients. BMC Cancer. 2016, 16:860. 10.1186/s12885-016-2905-X

8. Van Poznac C, Sauter NP: Clinical management of osteoporosis in women with a history of breast carcinoma. Cancer. 2005, 104:443-456. 10.1002/cncr.21201

9. Hoff AO, Gagel RF: Osteoporosis in breast and prostate cancer survivors . Oncology. 2005, 19:651-658.

10. NIH Consensus Development Panel on Osteoporosis Prevention, Diagnosis, and Therapy: Osteoporosis prevention, diagnosis, and therapy. JAMA. 2001, 285:785-795. 10.1001/jama.285.6.785

11. Siris ES, Miller PD, Barrett-Connor E, et al.: Identification and fracture outcomes of undiagnosed low bone mineral density in postmenopausal women: results from the National Osteoporosis Risk Assessment. JAMA. 2001, 286:2815-2822. 10.1001/jama.286.22.2815

12. Gardner MJ, Demetrakopoulos D, Shindle MK, Griffith MH, Lane JM: Osteoporosis and skeletal fractures . HSS J. 2006, 2:62-69. 10.1007/s11420-005-0137-8

13. Johansson H, Kanis JA, Oden A, Johnell O, McCloskey E: BMD, clinical risk factors and their combination for hip fracture prevention. Osteoporos Int. 2009, 20:1675-1682. 10.1007/s00198-009-0845-X

14. Johnell O, Kanis JA: An estimate of the worldwide prevalence and disability associated with osteoporotic fractures. Osteoporos Int. 2006, 17:1726-1733. 10.1007/s00198-006-0172-4

15. Rachner TD, Khosla S, Hofbauer LC: New horizons in osteoporosis . Lancet. 2011, 377:1276-1287. 10.1016/S0140-6736(10)62349-5

16. Kanis JA, Seeman E, Johnell O, Rizzoli R, Delmas P: The perspective of the International Osteoporosis Foundation on the official positions of the International Society for Clinical Densitometry. J Clin Densitom. 2005, 8:145-147.

17. Silva IM, Freitas DQ, Ambrosano GM, Bóscolo FN, Almeida SM: Bone density: comparative evaluation of Hounsfield units in multislice and cone-beam computed tomography. Braz Oral Res. 2012, 26:550-556.

18. Johnson CC, Gausden EB, Weiland AJ, Lane JM, Schreiber JJ: Using Hounsfield units to assess osteoporotic status on wrist computed tomography scans: comparison with dual energy X-ray absorptiometry. J Hand Surg Am. 2016, 41:767-774. 10.1016/j.jhsa.2016.04.016

19. Krappinger D, Roth T, Gschwentner M, Suckert A, Blauth M, Hengg C, Kralinger F: Preoperative assessment of the cancellous bone mineral density of the proximal humerus using CT data. Skeletal Radiol. 2012, 41:299-304. 10.1007/s00256-011-1174-7

20. Schreiber JJ, Anderson PA, Rosas HG, Buchholz AL, Au AG: Hounsfield units for assessing bone mineral density and strength: a tool for osteoporosis management. J Bone Joint Surg Am. 2011, 93:1057-1063. 10.2106/JBJS.J.00160 
21. Pickhardt PJ, Pooler BD, Lauder T, del Rio AM, Bruce RJ, Binkley N: Opportunistic screening for osteoporosis using abdominal computed tomography scans obtained for other indications. Ann Intern Med. 2013, 158:588-595. 10.7326/0003-4819-158-8-201304160-00003

22. Weber NK, Fidler JL, Keaveny TM, et al.: Validation of a CT-derived method for osteoporosis screening in IBD patients undergoing contrast-enhanced CT enterography. Am J Gastroenterol. 2014, 109:401-408. 10.1038/ajg.2013.478

23. Burke CJ, Didolkar MM, Barnhart HX, Vinson EN: The use of routine non density calibrated clinical computed tomography data as a potentially useful screening tool for identifying patients with osteoporosis. Clin Cases Miner Bone Metab. 2016, 13:135-140. 10.11138/ccmbm/2016.13.2.135

24. Floriano-Sanchez E, Rodriguez NC, Bandala C, Coballase-Urrutia E, Lopez-Cruz J: CYP3A4 expression in breast cancer and its association with risk factors in Mexican women. Asian Pac J Cancer Prev. 2014, 15:3805-3809. 10.7314/арjсp.2014.15.8.3805

25. Parada-Huerta E, Alvarez-Dominguez T, Uribe-Escamilla R, et al.: Metastasis risk reduction related with beta-blocker treatment in Mexican women with breast cancer. Asian Pac J Cancer Prev. 2016, 17:2953-2957.

26. Leon-Hernandez SR, Padilla EL, Algara AC, et al.: Relation of alcohol/tobacco use with metastasis, hormonal (estrogen and progesterone) receptor status and c-erbB2 protein in mammary ductal carcinoma. Asian Pac J Cancer Prev. 2014, 15:5709-5714. 10.7314/apjcp.2014.15.14.5709

27. Bandala C, Floriano-Sánchez E, Cárdenas-Rodríguez N, López-Cruz J, Lara-Padilla E: RNA expression of cytochrome P450 in Mexican women with breast cancer. Asian Pac J Cancer Prev. 2012, 13:2647-2653. 10.7314/apjcp.2012.13.6.2647

28. Guglielmi G, Muscarella S, Bazzocchi A: Integrated imaging approach to osteoporosis: state-of-the-art review and update. Radiographics. 2011, 31:1343-1364. 10.1148/rg.315105712

29. Cole SW, Sood AK: Molecular pathways: beta-adrenergic signaling in cancer . Clin Cancer Res. 2012, 18:1201-1206. 10.1158/1078-0432.CCR-11-0641

30. Batawil N, Sabiq S: Hounsfield unit for the diagnosis of bone mineral density disease: a proof of concept study. Radiography. 2016, 22:93-98. 10.2106/JBJS.J.00160 